\title{
Applicability Analysis of Semi-Network Operating System
}

\author{
Yin Sheng Zhang ${ }^{1}$ \\ ${ }^{1} \mathrm{PhD}$ Candidate of Electrical and Computer Engineering, University of British Columbia, Vancouver, Canada \\ Correspondence: Yin Sheng Zhang, University of British Columbia, Vancouver, Canada. Tel: 1-604-279-8620. E- \\ mail: cxo888@hotmail.com
}

Received: November 17, 2018

Accepted: November 28, $2018 \quad$ Online Published: January 27, 2019

doi:10.5539/cis. v12n1p93

URL: https://doi.org/10.5539/cis.v12n1p93

\begin{abstract}
Innovation of computing technology is either to improve the security and performance, or to improve the convenience of user operation of system, networking and device, but sometimes it's hard to get a very good balance between the two, in particular, the traditional localized OS and computing devices are inherently incompatible in some respects with today's network environment, therefore, although many innovations help to improve the safety, autonomy and controllability of system and device, but they are still always lacking the trustworthiness of users, one of the reasons is that their performance is lower than user's expectation, but their operation become more complicated than before.

The semi-network operating system is designed according to user's actual experience, which not only utilizes the shared attributes of network to increase the flexibility of OS, but also utilizes the stability and user-autonomy attributes of local platform ensure the base functions of OS, in addition, it can greatly reduce the burden on user's operation and maintenance of computing system, so which will be a trustworthy system architecture for users.
\end{abstract}

Keywords: base portion, expanded portion, Operating System Processing Unit (OSPU), semi-network OS

\section{Introduction}

Computing technology are always in the process of innovation, and new measures are constantly emerging, however, although the vast majority of measures can improve the autonomy, security and controllability of device and system, but there are still some measures that cannot obtain the trust and enthusiasm of users, one of the reasons is that their performance is lower than user's expectation, but their operation is more complicated than before.

The "semi-network OS" mentioned here refers to a concept of a new OS architecture that provides users with better autonomy, security and controllability.

\subsection{Incompatibility of Traditional OS and Today's Network Environment}

Some phenomena very clearly indicate the incompatibility of traditional localized OS and today's real network environment, which are:

Today, various application software can become network shared resources, but OS files running on fat client are still located outside network, then all system files of an OS can only be installed on a fat client device, which leads to fat client storage space being squeezed by a large amount of system files, and the performance of device is also forced to decrease.

Today, various client devices are inseparable from network, but the traditional localized OS is still always isolated from network, because of this isolation, the OS should rely on increasing application software to build connection between fat clients and network server, as well as protecting the security of fat client, which leads to very high stacking rate of application software in fat client device, and a lot of junk software downloaded from network can make fat client overwhelmed and offset the effects of technology advancement .

Today, all kinds of client devices regard network security as the top priority, but the traditional localized OS is also the biggest killer of network security, that's because it requires a fat client to open the door for software free installation, which provides a place to hide and a base to launch network attack for malware, such as network viruses, and then it itself becomes a victim of attacks from malware.

It should be noted here that the cloud OS is only a network application based on traditional localized OS, which 
cannot run on fat client platform and does not conform to the definition of "operating system", therefore, it is not the kind of OS that is traditionally defined.

\subsection{Bottom-Up Solution}

It is precisely because the fat client device can't get rid of the incompatibility between traditional localized OS and network environment ,the semi-network OS should provide a bottom-up solution, such as "expanded portion" of OS to create a network resources for system files, and to solve the problem of network sharing of system file; it replaces the free software installation platform of fat client with "Operating System Processing Unit"(OSPU) to solve the problem of fat clients to be cumulated by various junk software and malware; it uses the "base portion" of OS to create a local system resources that are fully adaptive to network environment, then this local system resource is integrated with the network system resource under the operation of "Operating System Processing unit" (OSPU), thereby realizing direct connection between OS and network without the need for application software to act as intermediary.

Such a solution not only avoids the excessive accumulation of applications software on fat client, but also makes malware such as viruses and hackers to lose their hiding place in fat client, and the fat client no longer needs to endlessly add application software to coordinate interaction with network server.

In addition, such a solution can also overcome the limitations of pure software OS.

The current mainstream OS, whether cloud OS or local OS, is simply a system program or application, once its software system is successfully installed on platform at one end of network, the software system can be run on that platform independently without the need for hardware components such as "Operating System Processing Unit" (OSPU) to participate, and there is also no need for close cooperation between client device and network server.

However, the semi-network OS must rely on a special hardware component to form an overall OS and rely on this hardware component to perform the running function, and this hardware component is "Operating System Processing Unit" (OSPU).

\section{Configuration of Semi-Network OS}

Semi-network OS (or semi-cloud OS) is a new system architecture of fat client with high autonomous, secure and controllable attributes for user to use, the reason why it is called "semi-network" is because its configuration mode and operation mechanism are based on local computing, which is completely different from the current popular so-called cloud OS that based on server computing, at the same time, it has to directly and partially rely on network resources to create operational flexibility and eliminate the harmful effects of malware on local platform, which is also completely different from the traditional localized OS that is isolated from network and relies on application software to establish contact with network.

The configuration of this new system architecture is: complete set of OS is divided into two parts by semi-network data technology, one is the "expanded portion" (network resources), and the other is "base portion" (client-end resources).

"Expanded portion" of semi-network OS is stored on network server to meet the diverse and mutable operating needs of fat clients.

"Base portion" of semi-network OS is stored in "Operating System Processing Unit" (OSPU) to ensure the stability of fat client platform operation.

The "Operating System Processing Unit" (OSPU) is a core hardware components located in fat client, acting as the regulation centre to ensure that the "base portion" can be safely rooted on specific fat client, "expanded portion" can be successfully downloaded to specific fat client, these two portions can be combined into a single seminetwork OS running on specific fat client, as well as managing the download and upload of application software and user data related to semi-network OS.

This new system architecture is not a cloud OS that based on server computing, nor is it a traditional OS that uses fat client as a permanent installation platform, but a new architecture of OS that gathers the advantages of two traditional OS and eradicates the disadvantages of two traditional OS, which not only make full use of network features to increase functional flexibility of fat clients, but also get rid of the shackles of network, and avoiding the poor fat client running caused by task over-crowding of server computing.

\section{Flexibility feature of Semi-Network OS}

The most important feature of the semi-network OS is its flexibility of operational function and file volume: a complete OS is divided into two portions, one is the "expanded portion" of semi-network OS (network resources), 
and the other one is the "base portion" (local resource) of semi-network OS, and the "expanded portion" is stored on network server, but it cannot run on network server, and it is used to meet the diversified operation requirements of fat client, and is downloaded according to the actual operation of the fat client.

The "expanded portion" of semi-network OS cannot be permanently stored in fat client, nor can it run on fat client independently, and it can only be run by a overall "semi- network OS" that integrating the "base portion" and "expanded portion" into one.

The "base portion" is stored in OSPU to ensure the stability of operation of fat client platform, where the OSPU is the core hardware component of fat client acting as the adjustment center and flexible mobile platform for seminetwork OS, which device is plugged in, the "base portion" of OS with user's personal settings will run on that device then, and the "expanded portion" of OS will also track the OSPU and downloads from server to that device, thus, the overall semi-network OS with personal settings will run on that device.

This is a new system architecture that runs on a fat client, but most of its system files are sourced from network shared resources, moreover, the semi-network OS is a system that includes hardware and software rather than a pure system software program, which has more opportunities and means to strengthen the autonomy, security and controllability of OS than traditional system with software alone, therefore, its running process is also a process of trusted computing.

\section{User-Focused Feature of Semi-Network OS}

In semi-network system architecture:

A. Because local computing is foundation, operating autonomy remains in hands of users, and the hardware component- OSPU can be independently and movably used on different devices by users, so this new new system architecture is more autonomous than the traditional model.

B. Because the fat client no longer needs hard disk to serve as a platform for free installation of system and application software (diskless), the system files of OS will come from network (expanded portion) and OSPU (base portion), and all system files are in a closed system and network channel, and third-party illegal intervention is strictly prohibited, that is, the mode of software free installation on fat client will be replaced by a "semi-network" mode, so the new system architecture is safer than traditional one.

C. Because the resources of OS at both ends of network have a unified control center-OSPU, and this control center (OSPU) is installed on fat clients at the discretion of user, so this new system architecture is more controllable than traditional model.

In semi-network system architecture, although most of system files of OS come from network server, but OS runs on fat client, and the control of system operation is still in hands of users, as a result, users do not need to change their operation habit formed before for traditional devices, so which is completely different from some innovations of localized OS, and is also completely different from some innovations of cloud OS.

This new system architecture aims to develop an OS that running on various fat clients and based on network sharing resource, so it will include a series of new mechanisms and new components, such as system programming, application release process, data encryption, and so on, as well as OSPU hardware components and network servers, this series of software programs and hardware components work together to build a complete semi-network OS.

Thus, it can be said that the semi-network OS is designed to eradicate some of deficiencies of traditional OS by trying to combine achievements of new and emerging networking technologies, coding technologies, chip technologies, to create new system architecture with great prospects and long-term vitality.

\section{Components of Semi-Network OS}

The semi-network OS consists of three core elements: a network platform (remote server), an pure software OS, and an OSPU, wherein the OSPU is a hardware component, which can be directly installed as a component on motherboard of fat client device, or can be made into a compact connector device for user to carry around, and these three core elements have hardware and software, which are indispensable, and the system software is only one of these core elements.

OSPU is a kind of mobile hardware component, which can be freely carried and installed by different users in different devices, and the "base portion" and "expanded portion" of semi-network OS will move with the OSPU, and once a user has mastered the OSPU, he will also master the entire semi-network OS that equipped with personality settings, that is, a small OSPU hardware can allow users to jump out of the limits of a personal computing device, when a device is connected to a OSPU, which will instantly becomes the user's personal device with special settings. 
The semi-network OS belongs to local computing, and its autonomy is easily grasped by users, now with OSPU, the autonomy of OS is easier than before to implement.

In this new system architecture, the software running on the fat client is either in a closed environment or downloaded from the server, and then the downloaded software temporarily runs in random-access memory of fat client, which only stores data currently being used, in this case, the malware cannot be permanently retained in fat client, and its hiding place and motivation of activity also disappear, which determines that the system architecture has strong security.

The OSPU is the only connection point between client platform and network server, and the only channel through which system data and user data flow across network. The OSPU is an exclusive hardware component that does not have the same hidden dangers of being illegally modified and forged as software programs, in addition, in the case of OSPU is freely kept and controlled by users, the network data being managed by OSPU is obviously easier, more targeted, and more accurate than network data being managed by various application software, and such implementation is easier to see the actual effect, therefore, such a system architecture has very reliable controllability.

\section{OSPU Installed in a Traditional Device}

The traditional device mentioned here refers to a computing device installed a traditional localized OS, and the device installed OSPU means that the semi-network OS will coexist with a traditional localized OS in a device, and user selects one of them to use, in this case, OSPU can add new features to traditional devices, which include:

\section{A. OSPU is installed in traditional computing device as a "security component".}

The existing mainstream OS is the biggest target of viruses, hackers and other malware attacks, and these mainstream OS are installed on free-installing client platform, so client platform naturally becomes the location where the malware is harboured, and the base where the network attack by malwares is initiated, thus, users expect more and more powerful security measures to join the operational process of their devices.

If the OSPU is installed on the device motherboard as a "security component" and enters market together with motherboard, then the OSPU provides the client device with another OS without malware threat as an option, which is equivalent to providing a new security function for client device.

\section{B. OSPU is installed in traditional computing device as an "capacity expansion component".}

Existing mainstream OS always adapt to the ever-changing network environment by means of more and more additional applications, which makes the storage space in client device occupied by too much extra data.

The large amount of extra data makes computing power of client device greatly squeezed, and users expect to reduce the impact of this extra data on capacity and computing power of client device.

If the OSPU hardware device is installed on the device motherboard as a "capacity expansion component" , and enters market together with device motherboard, then the OSPU provides an alternative OS that combines local and network resources for client device, which is equivalent to providing additional data storage capacity and computing power for client devices.

\section{OSPU is installed in a traditional computing device as a "system backup component".}

The existing mainstream OS is usually the only OS in the client device, lacking the ability to cope with sudden system failures, once system is down, the device has to stop running. Users expect their computing devices to run more reliably and reliably, and expect their computing devices to be equipped with backup systems to prevent accidental system failure.

If the OSPU hardware device is installed on device motherboard as a "system backup component", and enters market together with device motherboard, the OSPU provides another option for client device to get rid of local platform constraints, which is equivalent to providing client device with an emergency measure to sudden failure of localized OS.

\section{Conclusion}

The unique advantage of a semi-network OS is that the actual autonomy, security and controllability it brings to user is significantly higher than the traditional localized OS, so that users will definitely feel it trustworthy, which is reflected in the following points:

A. The semi-network OS aggregates the advantages of localized $O S$ and cloud $O S$, and also eliminates the defects of the two traditional OS, which fully utilizes the features of network to increase the functional flexibility of fat client and in the meantime get rid of the constraints of network, such as that the computing platform being set on 
fat client can avoid the overcrowding of server computing tasks, and also ensures the smooth running of fat client.

B. Although most of system files of semi-network OS are from network server, but it runs on fat client, the control of system is still in the hands of user, so which does not have a very drastic effect on operating habits that the user has previously formed, that is, users can get an easier and safer operating experience without changing the habits of operating traditional device that previously formed, for example, they can remove the burden of system installation, system maintenance, and system security measures setting.

C. The semi-network OS can also coexist with a traditional localized $O S$ in a device for users to selectively use, which can eliminate the user's fear and resistance to change habits, give the traditional system a new life, and also increases the application path of computing devices and make local computing more secure and reliable.

\section{References}

Andy, C., Bavier, Mic Bowman, Brent, N., Chun, David, E., ... Mike, W. (2004). Operating Systems Support for Planetary-Scale Network Services. Retrieved from http://pages.cs.wisc.edu/ bart/739/papers/planet2004.pdf

Brian, N., Bershad, S. S., Przemyslaw, Pardyak, Emin G“un Sirer, Marc E. Fiuczynski, ... Susan J. E. (2004). Extensibility, Safety and Performance in the SPIN Operating System. Retrieved from https://cseweb.ucsd.edu/ savage/papers/Sosp95.pdf

Robert, R., Jonathon, D., Pramod, S., Daniel, G., Mike, H., ... Jay, L. (2007). The Flexlab Approach to Realistic Evaluation of Networked Systems. Retrieved from https://www.cs.utah.edu/flux/papers/flexlab-nsdi07.pdf

\section{Copyrights}

Copyright for this article is retained by the author(s), with first publication rights granted to the journal.

This is an open-access article distributed under the terms and conditions of the Creative Commons Attribution license (http://creativecommons.org/licenses/by/4.0/). 